\title{
On a mathematical model arising in MHD perturbed equilibrium for Stellarator devices. A numerical approach
}

\author{
J.I. Díaz \\ Dept. of Matemática Aplicada \\ Fac. de Matemáticas, UCM \\ 28040 Madrid, Spain \\ ildefonso.diaz@mat.ucm.es
}

\author{
P. Galán del Sastre and J.F. Padial \\ Dept. of Matemática Aplicada \\ E.T.S. de Arquitectura, UPM \\ 28040 Madrid, Spain \\ pedro.galan@upm.es jf.padial@upm.es
}

\begin{abstract}
We consider a mathematical model related to the stationary regime of a plasma of fusion nuclear, magnetically confined in a Stellarator device. Using the geometric properties of the fusion device, a suitable system of coordinates and averaging methods, the mathematical problem may be reduced to a two dimensional free boundary problem of nonlocal type, where the corresponding differential equation is of the Grad-Shafranov type. The current balance within each flux magnetic gives us the possibility to define the third covariant magnetic field component with respect to the averaged poloidal flux function. We present here some numerical experiences and we give some numerical approach for the averaged poloidal flux and for the third covariant magnetic field component.
\end{abstract}

Keywords-Mathematical model; Grad-Safranov equaiton; equilibrium; rearrangement; fix point; Stellarator

\section{INTRODUCTION}

We present here a short survey on some mathematical results and some numerical experiences on a non-local two dimensional free boundary problem modeling the magnetic confinement of a plasma in a Stellarator device. Although we shall explain later the modeling, let us mention now that the problem (such as it was introduced in [1]. See also [2]) can be formulated in form of a free boundary problem: let $\Omega$ be an open, bounded, regular set of $\mathbb{R}^{2}$, and let $\lambda>0, F_{v}>$ $0, a, b \in L^{\infty}(\Omega), b>0$ a.e. in $\Omega$. Given $\gamma<0$, the problem is to find $u \in H^{1}(\Omega) \cap L^{\infty}(\Omega)$ and $F \in \mathcal{C}^{0}(\mathbb{R} ;[0, \infty))$ such that $F(s)=F_{v}$ for any $s \leq 0, F^{2} \in W_{l o c}^{1, \infty}(\mathbb{R})$ and $(u, F)$ satisfies the following inverse problem:

$$
\left(\mathcal{P}_{I}\right) \begin{cases}-\Delta u=a F(u)+\frac{1}{2}\left(F^{2}\right)^{\prime}(u)+\lambda b u_{+} & \text {in } \Omega, \\ u=\gamma & \text { on } \partial \Omega, \\ 0=\int_{\{x \in \Omega: u(x)>t\}}\left(\frac{1}{2}\left(F^{2}\right)^{\prime}(u)+\lambda b u_{+}\right) d x & \\ \forall t \in\left[\operatorname{essinf}_{\Omega} u,{\left.\operatorname{ess} \sup _{\Omega} u\right] .}\right. & \end{cases}
$$

where, for the sake of simplicity in the exposition, we have taken the pressure term equal to $p(s)=\frac{\lambda}{2} s_{+}^{2}$. Notice that we are not assuming the continuity of the solution. This is why we are using the notation $\operatorname{essinf}_{\Omega} u$ and $\operatorname{ess}_{\sup } u$ typically of elements in $L^{\infty}(\Omega)$, instead of $\min _{\Omega} u$ and $\max _{\Omega} u$ (as it is made for the case of continuous solutions).

In the sequel, we will refer to the family of integral identities, for any $t \in\left[\operatorname{essinf}_{\Omega} u, \operatorname{ess}_{\sup _{\Omega}} u\right]$, stated in $\left(\mathcal{P}_{I}\right)$

$$
\int_{\{x \in \Omega: u(x)>t\}}\left(\frac{1}{2}\left(F^{2}\right)^{\prime}(u(x))+\lambda u_{+}(x) b(x)\right) d x=0
$$

as the Stellarator Condition.

We point out that many other nonlocal problems arise in other problems related to the magnetic confinement of a plasma in which the above Stellarator condition is replaced by other conditions verified in the interior of the magnetic surfaces (see, e.g. the exposition, and its many references, of the paper [3]).

Problem $\left(\mathcal{P}_{I}\right)$ arises in the study of the magnetic confinement of a plasma in a Stellarator. The Stellarators are a class of toroidal plasma confinement devices alternative to the Tokamaks. The currents producing poloidal magnetic fields in Stellarators flow in external conductors allowing a wider range of magnetic configurations than those found in Tokamaks. The geometry of these magnetic configurations is very important since it is directly related to the stability and transport of the plasma.

The modelling of the problem starts by considerating the ideal magnetohydrodynamics (MHD) as the most basic single fluid model for determining the macroscopic properties of a plasma. The static MHD equilibrium is determined by the system

$$
\begin{aligned}
\nabla p & =\mathbf{J} \times \mathbf{B} \\
\nabla \times \mathbf{B} & =\mathbf{J} \\
\nabla \cdot \mathbf{B} & =0
\end{aligned}
$$

where $p$ is the pressure, $\mathbf{B}$ the magnetic field and $\mathbf{J}$ the current 
density. From (2) it follows that

$$
\begin{aligned}
\mathbf{B} \cdot \nabla p & =0 \\
\mathbf{J} \cdot \nabla p & =0 .
\end{aligned}
$$

Then the pressure is constant on each magnetic surface. If such a surface lies in a bounded volume of space and has no edges and if neither $\mathbf{B}$ nor $\mathbf{J}$ vanish anywhere on it then by a well-known theory due to Alexandroff and Hopf it must be a toroid (i.e. a topological torus). Since the magnetic field lines are tangent to toroidal nested surfaces, it is useful to introduce a set of new toroidal coordinates $(\tilde{\rho}, \tilde{\theta}, \tilde{\phi})$, such that: $\tilde{\rho}=$ $\tilde{\rho}(x, y, z)$ is an arbitrary function which is constant on each nested toroid and $\tilde{\theta}=\tilde{\theta}(x, y, z)$ is the poloidal angle which is constant on any toroidal circuit but changes by $2 \pi$ over a poloidal circuit. The toroidal angle $\tilde{\phi}$ is defined analogously but interchanging the words poloidal by toroidal.

There are several special choices of $(\tilde{\rho}, \tilde{\theta}, \tilde{\phi})$ which are relevant for different purposes. Here we shall take the Boozer vacuum coordinates system [4], which are very useful for Stellarators since magnetic field lines becomes "straights" in the $(\tilde{\theta}, \tilde{\phi})$-plane. In what follows, for the sake of simplicity in the notation, we shall denote this set of coordinates by $(\rho, \theta, \phi)$.

For a vacuum configuration (i.e. without any plasma) the magnetic field $\mathbf{B}_{v}$ may be written in contravariant form as

$$
\mathbf{B}_{v}=B_{0} \rho \nabla \rho \times \nabla\left(\theta-t_{v}(\rho) \phi\right)
$$

where $t_{v}(\rho)$ is the so called vacuum rotational transform and $B_{0}$ is a positive constant. The covariant form of $\mathbf{B}_{v}$ is

$$
B_{v}=F_{v} \nabla \phi
$$

where $F_{v}$ is a constant (which customary is taken as positive). In practice, it is used the quasi-cylindrical-like Boozer set of coordinates $(\rho, \rho \theta, \phi)$ which have the usual near-axis behavior of the field components commonly used.

In contrast to Tokamaks, the Stellarators-type configurations are very complicated due to the fully three-dimensional nature of the device. To simplify the model to a two-dimensional problem different averaging methods were used: see [5] and [6]. Following the last reference we may decompose the magnetic field in terms of its toroidally averaged and rapidly varying parts. For a general function $f$ this decomposition takes the form $f=\langle f\rangle+\tilde{f}$ where

$$
\langle f\rangle:=\frac{1}{2 \pi} \int_{0}^{2 \pi} f d \phi .
$$

In our case, motivated by the set of coordinates $(\rho, \rho \theta, \phi)$, the natural way of doing that is $\frac{B^{i}}{D}=\left\langle\frac{B^{i}}{D}\right\rangle+\left(\frac{\tilde{B}^{i}}{D}\right)$ where $B^{i}$ are the contravariant components of the vacuum magnetic field, $i=\rho, \theta, \phi$, and $D$ is the Jacobian

$$
D=(\nabla \rho \times \rho \nabla \theta) \cdot \nabla \phi
$$

Using a suitable assumption (the Stellarator expansion hypothesis), in [6] showed that (4) leads to the equation

$$
\frac{\partial}{\partial \rho}\left(\rho\left\langle\frac{B^{\rho}}{D}\right\rangle\right)+\frac{\partial}{\partial \theta}\left(\left\langle\frac{B^{\theta}}{D}\right\rangle\right)=0,
$$

and thus to the existence of the averaged poloidal flux function $\psi=\psi(\rho, \theta)$ defined by

$$
\left\langle\frac{B^{\rho}}{D}\right\rangle=\frac{1}{\rho} \frac{\partial \psi}{\partial \theta} \quad \text { and }\left\langle\frac{B^{\theta}}{D}\right\rangle=-\frac{\partial \psi}{\partial \rho} .
$$

They also show that $\left\langle B_{\phi}\right\rangle$ is a function $\psi$ alone and the same for $\langle p\rangle$ (recall (5)). By introducing the usual notation

$$
F(\psi):=\left\langle B_{\phi}\right\rangle \quad \text { and } \quad p(\psi):=\langle p\rangle
$$

as in [6] we obtained a Grad-Shafranov type equation for $\psi$

$$
-\mathcal{L} \psi=a(\rho, \theta) F(\psi)+F(\psi) F^{\prime}(\psi)+b(\rho, \theta) p^{\prime}(\psi)
$$

where

$$
\begin{aligned}
\mathcal{L} \psi & :=\frac{1}{\rho}\left\{\frac{\partial}{\partial \rho}\left(a_{\rho \rho} \frac{\partial \psi}{\partial \rho}\right)+\frac{\partial}{\partial \rho}\left(a_{\rho \theta} \frac{\partial \psi}{\partial \theta}\right)\right. \\
& \left.+\frac{\partial}{\partial \theta}\left(a_{\theta \rho} \frac{\partial \psi}{\partial \rho}\right)+\frac{\partial}{\partial \theta}\left(a_{\theta \theta} \frac{\partial \psi}{\partial \theta}\right)\right\}
\end{aligned}
$$

with $a_{\rho \rho}(\rho, \theta):=\rho\left\langle g^{\rho \rho}\right\rangle(\rho, \theta), a_{\theta \theta}(\rho, \theta):=\frac{1}{\rho}\left\langle g^{\theta \theta}\right\rangle(\rho, \theta)$, $a_{\rho \theta}(\rho, \theta)=a_{\theta \rho}(\rho, \theta):=\left\langle g^{\rho \theta}\right\rangle(\rho, \theta)$ and where $\left\langle g^{i, j}\right\rangle$, $i, j=\rho, \theta$ are the averaged components of the Riemannian metric associated to the vacuum coordinates system (all those coefficients are $2 \pi$-periodic functions in $\theta$ ). The rest of the coefficients in (10) are given by

$$
a(\rho, \theta):=\frac{B_{0}}{\rho F_{v}}\left[\frac{\partial}{\partial \rho}\left(\rho^{2} t(\rho)\left\langle g^{\rho \rho}\right\rangle\right)+\frac{\partial}{\partial \theta}\left(\rho t(\rho)\left\langle g^{\rho \theta}\right\rangle\right)\right]
$$

and

$$
b(\rho, \theta):=\frac{F_{v}}{B_{0}}\left\langle\frac{1}{D}\right\rangle(\rho, \theta) .
$$

We remark that $b>0$ and that usually function $a$ does not have any singularity.

Equation (10) only holds on the (averaged) region occupied by the plasma. In order to get a global formulation as a free boundary problem we remark that in the vacuum region $\nabla p=$ 0 and so, using (7), a simpler analysis than before leads to the equation

$$
-\mathcal{L} \psi_{v}=a(\rho, \theta) F_{v} .
$$

Besides, it is clear that the free boundary (separating the plasma and vacuum regions) is a (toroidal) magnetic surface and, as $p=p(\psi)$, by normalizing, we can identificate the free boundary as the level line $\{\psi=0\}$, the plasma region as $\{\psi>0\}$ (and thus $\{p>0\}$ ) and the vacuum region by $\{\psi<0\}$ (and $\{p=0\}$ ). It is also well-known that the pressure cannot be obtained from the (MHD) system and some constitutive law must be assumed. Usually, for simplicity, it is assumed a quadratic law (see e.g. [7])

$$
p=\frac{\lambda}{2}\left[\psi_{+}\right]^{2}, \quad \psi_{+}=\max \{\psi, 0\}
$$


which is compatible with the above normalization. In order to extend the unknown $F(\psi)$ for negative values of $\psi$ we use again (7) and so we must find $\psi(\rho, \theta)$ and $F: \mathbb{R} \rightarrow \mathbb{R}_{+}$such that $F(s)=F_{v}$ for any $s \leq 0$, satisfying

$$
-\mathcal{L} \psi=a(\rho, \theta) F(\psi)+F(\psi) F^{\prime}(\psi)+\lambda b(\rho, \theta) \psi_{+}
$$

on any bidimensional open set (in the variables $(\rho, \theta)$ ) associated to a physical three-dimensional domain $\Omega^{3}$ (i.e. in the original cartesian variables $(X, Y, Z)$ ) containing in its interior the plasma region. If we take as $\Omega^{3}$ the interior of a vacuum magnetic surface, the construction of the Boozer coordinates implies that the associated open set in the $(\rho, \theta)$ variables becomes $\Omega=\{(\rho, \theta): \rho \in(0, R), \theta \in(0,2 \pi)\}$. The boundary of $\Omega^{3}$ is assumed to be a perfectly conducting wall and thus $\mathbf{B} \cdot \mathbf{n}^{\mathbf{3}}=0$ over $\Omega^{3}$, where $\mathbf{n}^{\mathbf{3}}$ denotes the outer normal vector to $\partial \Omega^{3}$. The averaging process implies that over the associated part of $\partial \Omega^{3}$ i.e. on $\Gamma_{R}=\{(R, \theta): \theta \in(0,2 \pi)\}$ we must have $\langle\mathbf{B}\rangle \cdot \mathbf{n}=0$, where $\mathbf{n}$ is the outer normal to $\Gamma_{R}$. From (8) we obtain that

$$
\frac{\partial \psi}{\partial \tau}=0 \quad \text { on } \Gamma_{R}
$$

with $\tau$ the unit tangent vector to $\Gamma_{R}$ : in other words

$$
\frac{\partial \psi}{\partial \theta}(R, \theta)=0
$$

which, for some (negative) constant $\gamma$, shows that

$$
\psi=\gamma \quad \text { on } \Gamma_{R}
$$

In contrast with Tokamak devices, it is not restrictive to assume $\gamma$ a priori given since in the vacuum region $\psi(\rho, \theta)=\psi_{v}(\rho)$. We have the relation $\psi_{v}^{\prime}(\rho)=C \rho t(\rho)$ with $t(\rho)$ and $C$ known. If, for instance, the Stellarator possesses a limiter then the location of the free boundary is well-determinated and so is the rest of the vacuum levels.

To complete the formulation of the problem under consideration we must add the Stellarator Condition imposing a zero net current within each flux magnetic surface. According to the averaging method by Hender and Carreras [6], this condition can be expressed (see e.g. [8]) as

$$
\int_{\{(\rho, \theta) \in \Omega: \psi>s\}}\left[F(\psi) F^{\prime}(\psi)+\lambda b(\rho, \theta) \psi_{+}\right] \rho d \rho d \theta=0
$$

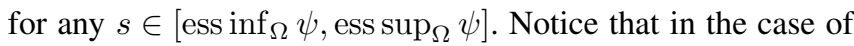
Stellarators this condition is set from the design of the external conductors, which differs from the usual condition of positive total current due to the inner toroidal current in the plasma for such configurations (see e.g. [7], [9] and [10]).

We point out that if we understand $(\rho, \theta)$ as the polar coordinates associated to a cartesian bidimensional space in the variables $(x, y)$, then the above set $\Omega$ is transformed into the ball $\hat{\Omega}:=\left\{(x, y): x^{2}+y^{2}<R^{2}\right\}$ and that if we define the identification $\hat{\psi}(x, y):=\psi(\rho, \theta)$ then, the boundary conditions (15), (16), (17) become

$$
\hat{\psi}=\gamma \text { on } \partial \hat{\Omega} .
$$

For the sake of simplicity in the exposition we have replaced, in (14), the elliptic operator $\mathcal{L}$ by the operator $\Delta$ (for a general treatment see [8]), and we replaced $(\rho, \theta)$ by the associated cartesian coordinate $x \in \Omega \subset \mathbb{R}^{2}$. As usual we have replace the physical notation of the unknown $\psi$ by the more common $u$ in mathematics. The equivalent problem to (14), (19), (18) but in Cartesian coordinate, is problem $\left(\mathcal{P}_{I}\right)$, what stated at the beginning of this section.

Before following, it seems interesting to mention the main differences of this problem with the model considered in the mathematical literature concerning the study of the confinement of a plasma in Tokamak devices (see e.g. [7], [9], [10], [11], [12], [13] and [14]). Due to the axisymmetry of the geometry, the unknown $u$ (the magnetic flux) in the Tokamak case may be written as direct function of the standard cylindrical coordinates system and so the operator $\mathcal{L}$ is the usual Laplacian operator $(\mathcal{L}=\Delta)$. More important seems to be the difference between the additional condition (18) (expressing the Stellarator condition of zero net current within each flux magnetic surface) and the Tokamak condition of positive total current

$$
\int_{\Omega}\left[F(u) F^{\prime}(u)+\lambda b u_{+}\right] d x=I
$$

for a prescribed $I>0$. Due to this fact, in the Tokamak case it seems not possible to determine the function $F$ unless if we have some extra information as, for instance, the value of the normal derivative of $u$ at $\partial \Omega$ (see [15] and its references). Then the mathematical model for Tokamaks assume a state law for $F$ similar to the pressure state law. It is usually assumed that $F(u) F^{\prime}(u)+\lambda b(x) u_{+}$can be written as $\mu c(x) u_{+}$for some $\mu \in \mathbb{R}_{+}$and $c \in L^{\infty}(\Omega)$ with $c>0$ in $\Omega$. The coefficient $a$ in (14) is intrinsic to Stellarator configurations and so it does not appear $(a \equiv 0)$ in the Tokamak model. We point out that terms of the form $a F(u)$ appear very often when modeling the Stellarator case (even if $(\rho, \theta)$ are taken in other different ways: see e.g. [5]). In conclusion, Stellarators lead to inverse type models, as $\left(\mathcal{P}_{I}\right)$, with $F$ unknown, in contrast with the Tokamak case where the final model corresponds to make formally $F \equiv 0$. Finally, due to the choice of the inverse coordinates $(\rho, \theta)$, the constant $\gamma$ appearing in (17) can be assumed to be known, which is not the case for the Tokamak model.

In practice, the ideal Stellarator Condition does not hold and a known current $j$ arises at the interior of each magnetic surface. In these sense, some studies on the stability of the equilibrium configuration, for the fixed boundary formulation, are already in the literature, see e.g. [16], where they assume $j(s)=j(1)\left(4 s^{2}-3 s^{4}\right)$ with $2 \pi j(1)$ is the total current inside the magnetic surface corresponding to $s=1$. This new condition is the so called current carrying Stellarator, which can be stated as

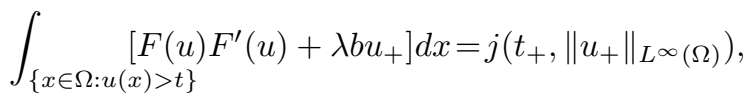


for all $s \in\left[\operatorname{ess} \inf _{\Omega} \psi, \operatorname{ess}_{\sup _{\Omega}} \psi\right]$. The mathematical study for current carrying Stellarator was done in [17].

Here, we consider a numerical approach for the problem $\left(\mathcal{P}_{I}\right)$, i.e., with $j \equiv 0$. To study problem $\left(\mathcal{P}_{I}\right)$, following [8], we will reformulate it in terms of a new problem $\left(\mathcal{P}_{*}\right)$, of nonlocal nature, where we replace the unknown function $F$, by a term involving the function $u$ and some functions associated to $u$ : its decreasing rearrangement $u_{*}$, and the relative rearrangement of $b$ with respect of $u, b_{* u}$. In order to justify it, we observe that by the Cavalieri Theorem, condition (18) can be written in terms of

$$
\int_{t}^{\infty}\left(\int_{\{u=t\}}\left[F(u) F^{\prime}(u)+\lambda b u_{+}\right] d x\right) d t=0
$$

for any $t \in\left[\operatorname{essinf}_{\Omega} u, \operatorname{ess}_{\sup _{\Omega}} u\right]$. Then differentiating (formally) with respect to $t$ we get that

$$
\int_{\{\psi=t\}}\left[F(u) F^{\prime}(u)+\lambda b u_{+}\right] d x=0
$$

for any $t \in\left[\operatorname{essinf}_{\Omega} u\right.$, $\left.\operatorname{ess}_{\sup _{\Omega}} u\right]$. And so, in particular,

$$
F(t) \frac{d F}{d t}(t)=\frac{\lambda t_{+} \int_{\{u=t\}} b(x) d \Gamma}{\int_{\{u=t\}} d \Gamma},
$$

for any $t \in\left[\operatorname{ess} \inf _{\Omega} u\right.$, $\left.\operatorname{ess}_{\sup _{\Omega}} u\right]$. A rigorous justification of the above formula was given in the papers [8].

In the next Section II, we show that if $u$ satisfies the family of conditions (1), then it is possible to express $F$ in terms of $u$, decoupling in this way the equation of such family of conditions. This fact gives us the key to reformulate the problem $\left(\mathcal{P}_{I}\right)$ as a non local problem $\left(\mathcal{P}_{*}\right)$ where we can eliminate the unknown function $F$. We begin introducing the notions of the decreasing and relative rearrangements, and some of their properties. The existence results of solution were proved in [8] and [17]. In Section III, we show a numerical approach to compute the solution of problem $\left(\mathcal{P}_{*}\right)$. We compute the numerical solution of $\left(\mathcal{P}_{*}\right)$, and consequently of $\left(\mathcal{P}_{I}\right)$, by using the finite element method combined with a fixed point algorithm. Similar approach was introduced in [18] for an helical model (in cylindrical coordinates). In [19] the authors introduced a numerical approximation of the relative rearrangement in one dimension and its application to a non local one dimensional problem, similar to problem $\left(P_{I}\right)$ but without term $F$. We compute the decreasing rearrangement and the relative rearrangement in two dimension and we obtain (numerically) the function $F$ and the distribution function of solution $u$ in terms of the associated decreasing and relative rearrangements.

\section{THE NON LOCAL PROBLEM $\left(\mathcal{P}_{*}\right)$}

We start recalling the notion of decreasing and relative rearrangement. Let $\Omega$ be a bounded and connected open measurable set of $\mathbb{R}^{2}$ (we assume a $2 \mathrm{~d}$-setting motivated by the physical modeling but the definitions and results that follows hold for any dimension $N>1$ ).

Definition 1: Given a general Lebesgue measurable function $u: \Omega \rightarrow \mathbb{R}$, the distribution function of $u$ is

$m_{u}(t):=\operatorname{meas}\{x \in \Omega: u(x)>t\}=|u>t|$ for any $t \in \mathbb{R}$

where by meas $\{E\}=|E|$ denotes the Lebesgue measure of any set $E \subset \mathbb{R}^{2}$.

The generalized inverse of $m_{u}$ is called the decreasing rearrangement of $u$ and is denoted by $u_{*}$. So, the function $u_{*}: \Omega_{*} \rightarrow \overline{\mathbb{R}}$ is such that

$$
u_{*}(s):=\inf \{t \in \mathbb{R}:|u>t|<s\}
$$

with $u_{*}(0)=\operatorname{ess}_{\sup _{\Omega}} u$ and $u_{*}(|\Omega|)=\operatorname{essinf}_{\Omega} u$ and where $\left.\Omega_{*}:=\right] 0,|\Omega|[$.

(The Lebesgue measure of the set $\{x \in \Omega: u(x)>t\}$ will be denoted by $|u>t|$ ).

It is well-know that the function $m_{u}(\cdot)$ is decreasing and right semicontinuous. We shall say that $u$ has a flat region at the level $t$ if meas $\{x \in \Omega: u(x)=t\}$ (denoting by $|u=t|$ ) is strictly positive (see e.g. [13] and [14] for more details about its definition and properties). We recall some properties: $u_{*}$ is decreasing, $u_{*}(0)=\left\|u_{+}\right\|_{L^{\infty}(\Omega)}=\operatorname{esssup}_{x \in \Omega} u(x), u_{*}$ and $u$ are equimeasurable, and the mapping $u \in L^{p}(\Omega)$ to $u_{*} \in L^{p}\left(\Omega_{*}\right)$ is a contraction for $1 \leq p \leq+\infty$. Moreover, if $u$ has not flat regions, then $m_{u}$ and $u_{*}$ are continuous and

$$
u_{*}\left(m_{u}(t)\right)=t \text { that is, } u_{*}^{-1}(t)=m_{u}(t)
$$

for all $t \in] \operatorname{ess} \inf _{\Omega} u$, $\operatorname{ess}_{\sup _{\Omega}} u[$. On the other hand, if $u \in$ $W^{1, p}(\Omega), 1 \leq p \leq+\infty$, then $u_{*} \in W_{l o c}^{1, p}\left(\Omega_{*}\right)$. Later, we will use these properties in the numerical computation of the functions $u_{*}$ and $m_{u}$.

Definition 2: Given a measurable function $u: \Omega \rightarrow \mathbb{R}$, and $b \in L^{p}(\Omega)$ with $1 \leq p \leq \infty$, we define the function $w: \Omega_{*} \rightarrow \mathbb{R}$ as

$$
w(s)=\int_{\left\{x \in \Omega: u(x)>u_{*}(s)\right\}} b(x) d x+\int\left(\left.b\right|_{\left\{x \in \Omega: u(x)=u_{*}(s)\right\}}\right)_{*}(\sigma) d \sigma .
$$

The relative rearrangement of $b$ with respect to $u$ is the function $b_{* u} \in L^{p}\left(\Omega_{*}\right)$ defined by

$b_{* u}(s):=\frac{d w(s)}{d s}=\lim _{\sigma \rightarrow 0} \frac{(u+\sigma b)_{*}(s)-u_{*}(s)}{\sigma}$ for all $s \in \Omega_{*}$

Notice that by this definition, if $u$ has not flat regions (implying $s-\left|u>u_{*}(s)\right|=0$ ) then

$$
b_{* u}(s):=\frac{d}{d s} \int_{\left\{x \in \Omega: u(x)>u_{*}(s)\right\}} b(x) d x \text { for all } s \in \Omega_{*} .
$$

Also, for any measurable function $u$, the mapping $b \in L^{p}(\Omega)$ to $b_{* u} \in L^{p}\left(\Omega_{*}\right)$ is a contraction for $1 \leq p \leq+\infty$ and in particular $\left\|b_{* u}\right\|_{L^{\infty}\left(\Omega_{*}\right)} \leq\|b\|_{L^{\infty}(\Omega)}$ (further details on the decreasing and relative rearrangement can be found, for instance, in [13], [14], [17], [20], , [21], [22], [23] and [24]). 
We introduce the following convex cone:

$$
V(\Omega)=\left\{v \in H^{1}(\Omega): \Delta v \in L^{\infty}(\Omega), v_{\left.\right|_{\partial \Omega}} \leq 0\right\} .
$$

We recall the existence result given in [8], [17] on the solution of problem $\left(\mathcal{P}_{I}\right)$.

Theorem 3: Suppose that $\gamma \leq 0$ and $\inf _{\Omega}|a|>0$. Then there exist $\Lambda_{1}, \Lambda_{2}>0$ such that if

$$
\lambda\|b\|_{L^{\infty}(\Omega)}<\Lambda_{1} \quad \text { and } \quad \Lambda_{2}<\inf _{\Omega}|a| F_{v}
$$

there is a couple $(u, F), u \in V(\Omega)$ and $F \in$ $W^{1, \infty}(]$ essinf $\sin _{\Omega} u, \operatorname{essup}_{\Omega} u[)$ solution of $\left(\mathcal{P}_{I}\right)$ satisfying also that meas $\{x \in \Omega: \nabla u(x)=0\}=0$.

Now, given $u \in W^{1, \infty}(\Omega)$, we define the real function $F_{u}$ : $\mathbb{R} \rightarrow \mathbb{R}_{+}$by

$$
F_{u}(t)=\left[F_{v}^{2}-\lambda \int_{0}^{t_{+}} s b_{* u}(|u>s|) d s\right]_{+}^{\frac{1}{2}}
$$

and we define the function $\mathcal{F}_{u}: \Omega \rightarrow \mathbb{R}$ as follows:

$$
\mathcal{F}_{u}(x):=\left[F_{v}^{2}-\lambda \int_{0}^{u_{+}(x)} s b_{* u}(|u>s|) d s\right]_{+}^{\frac{1}{2}} .
$$

Formally, the real function $F_{u}$ has been obtained taking the derivative with respect to $t$ on the Stellarator Condition (1). We set $\hat{m}=\inf _{\Omega} u$ and $M=\sup _{\Omega} u$ which are justified since $u \in L^{\infty}(\Omega)$. Then, we have

Lemma 4: Let $u \in W^{1, \infty}(\Omega)$ such that meas $\{x \in \Omega$ : $\nabla u(x)=0\}=0$. Then $\mathcal{F}_{u}(x)=F_{u}(u(x))$ for all $x \in \bar{\Omega}$.

Lemma 5: Assume $u \in V(\Omega)$ such that meas $\{x \in \Omega$ : $\nabla u(x)=0\}=0$ and $\min \left\{F_{u}(t): t \in[\hat{m}, M]\right\}>0$. Then

i) $F_{u} \in W^{1, \infty}(] \hat{m}, M[)$ and

ii) for almost every $t \in[\hat{m}, M]$, we have

$$
F_{u}(t) F_{u}^{\prime}(t)+\lambda u_{+} b_{* u}(|u>t|)=0 .
$$

\section{Moreover}

$$
F_{u}(u(x)) F_{u}^{\prime}(u(x))=-\lambda u_{+}(x) b_{* u}(|u>u(x)|) .
$$

Lemma 6: Let $u \in V(\Omega)$ such that meas $\{x \in \Omega: \nabla u(x)=$ $0\}=0$ and let $F_{u}$ given by (23). Assume that $\min \left\{F_{u}(t), t \in\right.$ $[\hat{m}, M]\}>0$. Then, for all $t \in[\hat{m}, M]$

$$
\int_{\{x \in \Omega: u(x)>t\}} F_{u}(u(x)) F_{u}^{\prime}(u(x))+\lambda u_{+}(x) b(x) d x=0 .
$$

See [17] for the proof. Notice that the above lemma tells us that given a function $u \in V(\Omega)$ without flat region (i.e. meas $\{x \in \Omega: \nabla u(x)=0\}=0)$ and defining $F_{u}$ as in (23), then the pair $\left(u, F_{u}\right)$ satisfies the Stellarator Condition (1). So, we reduce the original problem $\left(\mathcal{P}_{I}\right)$ to the non local problem: find the only one unknown $u$ for the problem

$\left(\mathcal{P}_{*}\right)\left\{\begin{array}{c}-\Delta u=a \mathcal{F}_{u}(x)+\lambda u_{+}\left[b(x)-b_{* u}(|u>u(x)|)\right] \text { in } \Omega, \\ u-\gamma \in H_{0}^{1}(\Omega) .\end{array}\right.$
Under the condition that $u$ has not flat regions we obtain the equivalence between both problems:

Theorem 7: Let $(u, F)$ be a solution of $\left(\mathcal{P}_{I}\right)$ given by Theorem 3 and assume that $\mathcal{F}_{u}(x)>0$, for all $x \in \bar{\Omega}$, then $u$ is a weak solution of the non local problem $\left(\mathcal{P}_{*}\right)$. Reciprocally, if $u$ is a weak solution of $\left(\mathcal{P}_{*}\right)$ such that $u$ has not flat region $(\operatorname{meas}\{x \in \Omega: \nabla u(x)=0\}=0)$ and such that $F_{u}>0$ in $\left[\operatorname{essinf}_{x \in \Omega} u, e \operatorname{essup} \operatorname{sen}_{x \in \Omega} u\right]$, then $\left(u, F_{u}\right)$ is a solution of $\left(\mathcal{P}_{I}\right)$ where $F_{u}$ is defined as in (23) and $F_{u} \in W^{1, \infty}(] \hat{m}, M[)$.

Remark 8: i) Notice that $u$ is the only one unknown of problem $\left(\mathcal{P}_{*}\right)$.

ii) We can verify that if $s \leq 0$ then $F_{u}(s)=F_{v}>0$ (it comes from (23)). If $u(x) \leq 0$ then $F_{u}(u(x))=F_{v}$.

iii) The assumption $\mathcal{F}_{u}(x)>0, x \in \bar{\Omega}$ for a given $(u, F)$ solution of $\left(\mathcal{P}_{I}\right)$ in Theorem 7 , it is not restrictive condition because of this will be true for every solution given by Theorem 3 .

iv) Theorem 7 allows us to work with $u$ as weak solution of $\left(\mathcal{P}_{*}\right)$ or $\left(u, F_{u}\right)$ as a weak solution of $\left(\mathcal{P}_{I}\right)$ indistinctly. Thus, in what follows, we only consider the above mentioned regularity of $\lambda$ small enough (see [8] and [17] for more details).

\section{NUMERICAL APPROXIMATION}

We compute the numerical solution of $\left(\mathcal{P}_{I}\right)$ using the finite element method combined with a fixed point algorithm. Let $D_{h}$ be a partition of $\Omega$ such that $D_{h}=\left\{Q_{i}\right\}_{i=1}^{N_{e}} \subset \bar{\Omega}$, where $Q_{i}$ are rectangles and $N_{e}$ the number of finite elements in the partition. Then the finite element subspaces $V_{h}$ is defined as

$$
V_{h}=\left\{v_{h} \in C(\bar{\Omega}): v_{h \mid Q_{i}} \in P_{1}\left(Q_{i}\right) \forall i=1,2, \ldots, N_{e}\right\}
$$

and $V_{h 0}=V_{h} \cap H_{0}^{1}(\Omega)$, where $P_{1}\left(Q_{i}\right)$ is the set of polynomials $\sum_{j} c_{j} p_{j}(x) q_{j}(y)$ where $p_{j}$ and $q_{j}$ are polynomials of degree $\leq 1$.

Let $u_{h}^{(0)} \in V_{h}$ such that $u_{h}^{(0)}-\gamma \in V_{h 0}$, then the discretized problem consists in solving, for any $k=0,1,2, \ldots$; find $u_{h}^{(k+1)} \in V_{h}$ such that $u_{h}^{(k+1)}-\gamma \in V_{h 0}$ and

$$
\left(\nabla u_{h}^{(k+1)}, \nabla v_{h}\right)=\left(g_{h}^{(k)}, v_{h}\right) \text { for all } v_{h} \in V_{h 0},
$$

where $g_{h}^{(k)} \in V_{h}$ is an approximation of the function $a F\left(u_{h}^{(k)}\right)+\frac{1}{2}\left(F^{2}\right)^{\prime}\left(u_{h}^{(k)}\right)+\lambda b\left(u_{h}^{(k)}\right)_{+}$.

Note that when solving problem (26), we first need to compute the function $g_{h}^{(k)}$ in the right hand side.

\section{A. Computing the function $g_{h}^{(k)}$}

The used algorithm is the following:

1) Start with a given function $u_{h}^{(0)} \in V_{h}$ (without flat region).

2) Step $k$. Given $u_{h}^{(k)}$ by (26)

Then, $u_{h}^{(k)}$ has not flat region. 

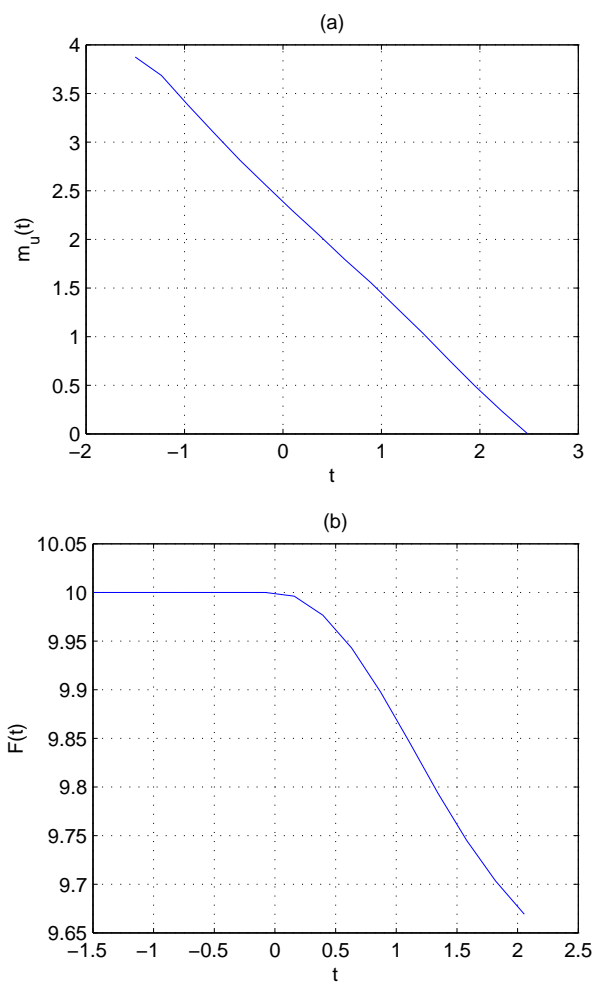

Figure 1. (a) Distribution function of averaged poloidal flux $m_{u}$; (b) third component of covariant form of the magnetic field $F$.

a) Obtain an approximation of the distribution function $m_{u_{h}^{(k)}}$. Let $T=\left\{t_{0}=\max _{\Omega} u_{h}^{(k)}>t_{1}>\right.$ $\left.\ldots>t_{h z}=0\right\}$ a mesh of interval $\left[0, \max _{\Omega}(u)\right]$. We sort the array of mapping of $u_{h}^{(k)}$ on $x$ in the mesh of $\bar{\Omega}$ and $m_{u_{h}^{(k)}}\left(t_{i}\right)=\left|u_{h}^{(k)}(x)>t_{i}\right| \approx$ $\sum_{\left\{x: u_{h}^{(k)}(x)>t_{i}\right\}}$ weight $(x)$ where the weighted function is taking accordingly either $x \in \partial \bar{\Omega}$ or $x \in \Omega$.

b) Obtain the decreasing rearrangement $\left(u_{h}^{(k)}\right)_{*}$. Since $u_{h}^{(k)}$ has not flat region, $\left(u_{h}^{(k)}\right)_{*}(\cdot)=m_{u_{h}^{(k)}}^{-1}(\cdot)$ (see (21)).

c) Obtain the relative rearrangement $b_{\left(u_{h}^{(k)}\right)}$. Since $u_{h}^{(k)}$ has not flat region, compute $b\left(u_{h}^{(k)}\right)$ following (22) by discrete integration and differentiation. First integrating $b$ over $\left\{x \in \Omega: u_{h}^{(k)}(x)>\left(u_{h}^{(k)}\right)_{*}(s)\right\}$ for all $s \in$ $\Omega_{*}$ and finally differentiating with respect to $s$, with $\left(u_{h}^{(k)}\right)_{*}(s) \in T$.

d) Compute $F:=F_{u_{h}^{(k)}}$. By trapezoidal integration role, following (23) for any $t \in T$.

e) Obtain $g_{h}^{(k)}$ which was defined in (26). We derivate $\frac{1}{2} F_{u_{h}^{(k)}}^{2}$ in $T$, and then, by lineal interpolation of $\left(\frac{1}{2} F_{u_{h}^{(k)}}^{2}\right)^{\prime}$ and $F_{u_{h}^{(k)}}$ on the array of mapping of $u_{h}^{(k)}$
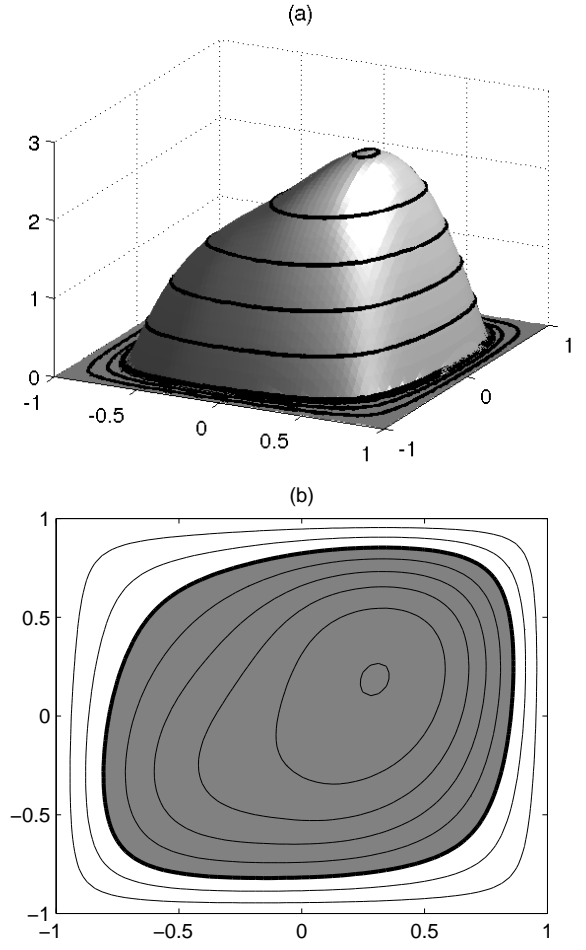

Figure 2. (a) Averaged poloidal flux $u$ obtained by the numerical scheme; (b) and level set of the solution at the interior of its free boundary (i.e. on the plasma region, $u>0$, in grey).

on $x$ in the mesh of $\bar{\Omega}$; we compute $g_{h}^{(k)}$ on $\Omega$.

f) Solve discretized problem (26) by Conjugate Gradient (see e.g. [25]).

3) Stopping criterion.

¿From this algorithm, we can obtain as intermediate step, the distribution function $m_{u}$ and the function $F$ (see Fig. 1). The representation of the free boundary and other level lines of the solution is made in Fig. 2.

\section{NUMERICAL RESULTS}

In this work, the authors have implemented their own code using the $\mathrm{C}$ language to solve the partial differential equation by using the finite element method, as well as for the determination of distribution function of averaged poloidal flux.

We consider a test problem with $\Omega=[-1,1] \times[-1,1]$, $\gamma=-1.5, \lambda=1.4, F_{v}=10$ and

$$
\begin{aligned}
& a(x, y)=1.5+\sin (\pi x) \sin (\pi y) \\
& b(x, y)=5\left(1+\frac{2}{\pi} \arctan (10 x)\right) .
\end{aligned}
$$

The fix point is started using

$$
u_{0}(x, y)=-1.5-3\left(x^{2}-1\right)(x-1)\left(y^{2}-1\right) \text {. }
$$


The partition $D_{h}$ used to solved problem (26) by the finite element method consists in a regular rectangular mesh with $h=\frac{1}{32}$, i.e., 4096 elements and 4225 nodes. The associated linear system to (26) is solved using Conjugate Gradient. The CPU time consumed to compute the full algorithm is less than one second on an Intel Core i7 at $2.67 \mathrm{Ghz}$ processor.

Note that the choice of these data functions is only for testing purposes of the numerical scheme. In Fig. 2 we show the solution obtained by the numerical approach, that carries out less than one second of CPU time. For a real Stellarator the numerical approach only requires to use new data coming from equations (11) and (12) that depend one the geometry of a particular Stellarator device. The obtaining of those associated data functions (related to Boozer coordinate system) could be quite complex. Nevertheless, we can check that in any case, we obtained for the approximating solution the regularity given by the theoretical studies, when the regularity of the data is as in the assumptions of existence result or close to them. For large data $\lambda, b, a, F_{v}$ (in the sense of Theorem 3), we don't have convergence of the numerical approach.

\section{FUTURE WORK}

The methodology concerning the numerical results of this work can be applied in many different contexts. In particular it could be applied to the case of some parabolic version of the problem (see [26] and [27]), where even time implicit schemes could be considered due to the fast convergence of the algorithm of the stationary model. In another context, nonlocal formulations arising when the confinement of the plasma takes place in Tokamaks (see e.g. the exposition made in [3], [28] and [23]) could also be handled with this methodology. Finally, it is clear that enhancements on the code that could require a more powerful computing platform and the possibilities of distributing the execution of the problem in parallel or distributed tasks could be designed in the light of similar works in the literature on parallel computing for nonlinear elliptic partial differential equations (see, e.g. [29]).

\section{ACKNOWLEDGMENT}

The research of J.I. Díaz has received funding from the ITN FIRST of the Seventh Framework Programme of the European Community (grant agreement num. 238702) and it was partially supported by the Research Group MOMAT (Ref. 910480), UCM and the project MTM2011-26119 (DGISPI, Spain). P. Galán's research has been partially funded by grant CGL2007-66440-C04-01 (MEC, Spain). The research of J.F. Padial was supported by the project MTM2011-26119 (DGISPI, Spain).

\section{REFERENCES}

[1] J.I. Díaz, Modelos bidimensionales de equilibrio magnetohidrodinámico para Stellarators, Informe \#2. CIEMAT Repports. Madrid, July 1992.
[2] J.I. Díaz, Un problema de frontera libre en el estudio del equilibrio magnetohidrodinámico de un plasma en una configuración Stellarator, in Modelos matemáticos en Física de Plasmas (J.I. Díaz y A.Galindo eds.), Memorias de la RAC, Tomo XXX, 73-132, 1995.

[3] S. Semenzato, R. Gruber and H.P. Zehrfeld, "Computation of symmetric ideal MHD flow equlibria", Computer Physics Reports, Vol. 1, 7\&8, 389-425, 1984.

[4] A.H. Boozer, "Establishment of magnetic coordinates for given magnetic field", Phys. Fluids, 25, 3, pp. 520-521, March 1982.

[5] J.M. Greene and J.L. Johnson, "Determination of Hydromagnetic Equilibria”, Phys. Fluids 27, pp. 2101-2120, 1984.

[6] T.C. Hender and B.A. Carreras, "Equilibrium calculation for helical axis Stellarators", Phys. Fluids, 27, pp. 2101-2120, 1984.

[7] R. Temam, "A nonlinear eigenvalue problem: equilibrium shape of a confined plasma”, Arch. Rational Mec. Anal., 60, pp. 51-73, 1975.

[8] J.I. Díaz and J.M. Rakotoson; "On a non local stationary free-boundary problem arising in the confinement of a plasma in a Stellarator geometry", Archive for Rational Mechanics and Analysis 134, pp. 53-95, 1996.

[9] J. Blum; Numerical simulation and optimal control in plasma physics, John Wiley \& Sons, New York, 1989.

[10] R. Temam, "Remarks on a Free Boundary Value Problem Arising in Plasma Physics", Comm. in Partial Differential Equations, 2 (6), pp. 563-585, 1977.

[11] H. Berstycki and H. Brezis, "On a free boundary problem arising in plasma physics”, Nonlinear Anal., 4, pp. 415-436, 1980.

[12] A. Friedman, Variational principles and free-boundary problems, John Wiley and Sons, New York, 1982.

[13] J. Mossino and R. Temam, "Directional derivative of the increasing rearrangement mapping and application to a queer differential equation in Plasma physics”, Duke Math. J., 48, pp. 475-495, 1981.

[14] J. Mossino and J.M. Rakotoson, "Isoperimetric inequalities in parabolic equations", Annalli della Scuola Normale Superiore de Pisa, Serie IV, 13, pp. 51-73, 1986.

[15] E. Beretta and M. Vogelius, "An inverse problem originating from Magnetohydrodynamics II: the case of the Grad-Shafranov Equation", Indiana University Mathematics Journal, 41 (4), pp. 1081-1117, 1992.

[16] W. A. Cooper; Global External Ideal Magnetohydrodynamic Instabilities in Three-dimensional Plasmas, Theory of Fusion Plasmas, Proc. of the Joint Varenna-Laussane Workshop, Edit. Compositori, Bologna 1990.

[17] J.I. Díaz, J.F. Padial and J.M. Rakotoson, "Mathematical treatment of the magnetic confinement in a current carrying Stellarator", Nonlinear Analysis Theory Methods and Applications 34, pp. 857-887, 1998.

[18] A. Bermúdez and M.L. Seoane; "Numerical solution of a non local problem arising in plasma physics", Mathematical and Computer Modelling, 27 (5), pp. 45-59, 1998.

[19] J.M. Rakotoson and M. L. Seoane, "Numerical approximations of the relative rearrangement. Applications to some non local problems", M2AN, 34 (2), pp. 477-499, 2000.

[20] J. Mossino, Inégalités isoperimétriques. Collection "Travaux en cours", Herman Paris 1984

[21] J.M. Rakotoson, "Some properties of the relative rearrangement", $J$. Math. Anal. Appl., 135, pp. 488-500, 1988.

[22] J.M. Rakotoson, "Strong continuity of the relative rearrangement maps and application to a Galerkin approach for non local problems", Applied Math. Letters, 8 (6), pp. 61-63, 1995.

[23] J.M. Rakotoson, "Galerkin approximation, strong continuity of the relative rearrangement and application to plasma physics equations", Diff. and Integral Equations, 12 (1), pp. 67-81, 1999.

[24] J.M. Rakotoson, Réarrangement Relatif: Un instrument d'estimations dans les problèmes aux limites, Mathématiques et Applications, SMAI, Springer, Paris 2008.

[25] P.G. Ciarlet, Intorduction à l'analyse numérique matriciaelle et à l'optimisation, Masson, Paris 1990.

[26] J.I. Díaz, M.B. Lerena and J.F. Padial, On a nonlocal quasilinear parabolic model related to a current-carrying Stellarator, Nonlinear Analysis: Real World Application, 3 (2002), 503-514.

[27] J.I. Díaz, M.B. Lerena, J.F. Padial and J.M. Rakotoson, An ellipticparabolic equation with nonlocal term for transient regime of a plasma in Stellarator, J. Differential Equations, 198 (2004), 321-355.

[28] J. Mossino, "A Priori Estimates for a Model of Grad Mercier Type in Plasma Confinement”, Applicable Analysis, 13, pp. 185-207, 1982.

[29] A. Quarteroni and A. Valli, Domain Decomposition Methods for Partial Differential Equations. Clarendon Press Oxford 1999. 